\title{
REPRODUÇÃO DE Meloidogyne incognita E CARACTERIZAÇÃO FENOLÓGICA DE ACESSOS DE FIGUEIRA
}

\author{
Ester Alice Ferreira ${ }^{1 *}$,Sônia Maria de Lima Salgado', Guilherme Augusto Teixeira Tassone ${ }^{1}$, Elisângela Aparecida \\ Silva², Ana Isabela Sales Bueno ${ }^{1}$ \\ 1 Universidade Federal de Lavras - C.P. 176 Campus UFLA Lavras MG. \\ ${ }^{2}$ Instituto Federal do Sudeste de Minas Gerais, Campus de Barbacena. Rua Monsenhor José Augusto, São José CEP 36205018 - \\ Barbacena, MG. \\ ${ }^{1 *}$ Autor para correspondência: ester@epamig.br
}

\begin{abstract}
RESUMO: A cultivar 'Roxo de Valinhos', embora altamente suscetível ao nematoide de galhas, um dos principais problemas fitossanitários da cultura, é predominante nos plantios de figueira no Brasil. Para atender a necessidade de diversificação do material genético de figueira este trabalho teve como objetivo avaliar o ciclo vegetativo de onze acessos de figueira e a reprodução de Meloidogyne incognita neste material. As figueiras pertencentes ao Banco de Germoplasma do Núcleo de Produção de Mudas da Coordenadoria de Assistência Técnica Integral (CATI) foram avaliadas nas seguintes fases fenológicas: pontas verdes; alongamento dos ramos; desenvolvimento e maturação do fruto. Na avaliação da hospedabilidade ao nematoide, mudas dos mesmos acessos de figueira foram inoculadas com 5.700 ovos de $M$. incognita em sacos de polietileno em casa de vegetação. Após seis meses foram avaliados o número de ovos, número de juvenis no segundo estádio, número de galhas, população total (ovos + Juvenis do segundo estádio) por grama de raízes; fator de reprodução e massa fresca de raiz. A duração média do ciclo fenológico dos acessos foi de 226 dias e em todos os acessos ocorreu alta reprodução do $M$. incognita. Assim, podem ser classificados com suscetibilidade alta e estatisticamente igual à cv. Roxo de Valinho.
\end{abstract}

PALAVRAS-CHAVE: Ficus carica L.. Fenologia. Nematoide das galhas.

\section{REPRODUCTION OF Meloidogyne incognita AND PHENOLOGICAL CHARACTERIZATION IN FIG PLANTS ACCESS.}

\begin{abstract}
Roxo de Valinhos' is the prevailing cultivar in fig plantations in Brazil even though being highly susceptible to root-knot nematode, the major phytosanitary problem of fig tree culture. In order to attend the need to diversify fig tree genetic material, this work aimed to evaluate the vegetative cycle of eleven fig accessions and the reproduction of Meloidogyne incognita in this material. Fig trees from Germplasm Bank of Center of Integral Technical Assistance (CATI) - Seedling Production Section; were evaluated in the following phenological phases: unfolded leaves; branches growth; fruit development and its maturation. In assessment of nematode hospitability, fig seedlings from same eleven accessions were inoculated with 5,700 eggs of $M$. incognita in polyethylene bags in a greenhouse. After six months they were evaluated by: number of eggs, juveniles, second-stage of galls, total population (eggs and second-stage juveniles) per gram of roots; reproduction factor and fresh weight of roots. The average duration of the phenological cycle of the eleven fig tree accessions was 226 days and all plants presented high reproduction $M$. incognita. Thus, they can be classified with high and statistically equal susceptibility to cv. Roxo de Valinhos.
\end{abstract}

KEY WORDS: Ficus carica L.. Phenology. Root-knot nematode. 


\section{INTRODUÇÃO}

A figueira (Ficus carica L.) é uma das frutiferas mais tradicionais do mundo e vem sendo cultivada com sucesso há vários séculos no Brasil que atualmente é o oitavo maior produtor mundial. 0 figo se destaca ainda entre as frutas temperadas brasileiras que são exportadas, ocupando a terceira posição, atrás somente da uva e maçã e projetando o Brasil como maior produtor e principal exportador de figos do hemisfério sul, tendo como principal vantagem a produção na entressafra dos maiores produtores e consumidores de figos (IBGE, 2010; Fachinello, 2011; FAO, 2012).

A 'Roxo de Valinhos' é a cultivar mais plantada no Brasil, pela sua rusticidade, elevado vigor e produtividade, boa adaptação a podas drásticas e cujos frutos podem ser utilizados tanto para 0 consumo in natura quanto para 0 processamento (Maiorano et al., 1997). Entretanto, apesar de suas boas características, o risco deste monocultivo, tem contribuído para a vulnerabilidade da ficicultura a problemas fitossanitários, além de limitar o potencial de comercialização da fruta. A busca por novos materiais genéticos de figueira no Brasil tem sido dificultada pela ausência de programas de melhoramento via métodos convencionais, devido à dificuldade de obtenção de plantas oriundas de fusão gamética pela ausência da vespa Blastophaga psenes, responsável pela polinização natural (Ferreira et al., 2009).

Neste cenário, a introdução de novas cultivares é considerada a principal opção para diversificação da ficicultura brasileira. Essa introdução tem sido feita por instituições públicas a exemplo da CATI - Coordenadoria de Assistência Técnica Integral que possui um banco de germoplasma com acessos de diferentes procedências. A diversidade genética de alguns acessos mantidos pela CATI foi avaliada por Val et al. (2013) onde foram encontrados dez genótipos e duas sinonímias no material estudado.

Além da diversidade genética, a caracterização fenológica dos acessos de um banco de germoplasma é essencial no conhecimento do banco, e pode ser considerada a etapa inicial no estudo das fases de desenvolvimento das plantas. A fenologia baseiase nas observações dos eventos visíveis do ciclo de vida (fenofases) da planta, que são respostas às forças seletivas bióticas e abióticas, bem como da interrelação entre as fases caracterizadas por esses eventos (Lieth, 1974). A organização das datas proporciona informações ecológicas importantes sobre a duração média das diferentes fenofases das plantas em uma área, e sobre o local e as diferenças determinadas pelo clima nas datas de início dessas fases (Larcher, 2000). 0 conhecimento das fases fenológicas é imprescindível na definição das práticas de manejo necessárias ao cultivo da figueira, como demostrado por Souza et al. (2009), inclusive no manejo da produção de figos verdes (Gonçalves et al., 2006; Nienow et al., 2006).

Diversos trabalhos têm apontado o nematoide de galhas, especialmente $M$. incognita, como o principal fitonematoide parasita de plantas em pomar de figo. Em levantamento realizado em frutiferas na região noroeste do Estado do Paraná, aproximadamente 50\% das amostras encontrava-se parasitadas por diferentes gêneros de fitonematoides, sendo Meloidogyne, um dos mais frequentes, apresentando risco a fruticultura da região (Dias-Arieira et. al., 2010). A presença do nematoide das galhas foi observada por Medina et al. (2013) em todas as amostras de raízes de figueira de diferentes pomares do Estado do Rio Grande do Sul, Sul do Brasil. Nesse trabalho os autores identificaram duas espécies do nematoide das galhas em figueira, M. incognita com os fenótipos (Est/Mdh) I1/N1, I2/ $\mathrm{N} 1, \mathrm{~S} 1 / \mathrm{N} 1$ e ou S2/N1, em todos os pomares; e $M$. javanica J2/N1 associada a $M$. incognita I1/N1 em apenas um pomar. Em trabalho anterior, Medina et al. (2006) detectaram M. incognita em $80 \%$ das amostras coletadas em pomares no Estado de São Paulo, maior produtor nacional, e em $92 \%$ dos pomares amostrados no Rio Grande do Sul (Gomes et al., 2009).

Entre as principais táticas de manejo a serem adotadas para 0 controle eficiente e econômico da meloidoginose em figueira destaca-se 0 material genético resistente. Porém, Medina et al. (2013) citam que a utilização de porta-enxertos tem sido restrita pela indisponibilidade de materiais tolerantes ou resistentes ao nematoide de galhas. A resistência da planta está na capacidade de restringir ou prevenir a multiplicação do nematoide (Moura, 1997), consequentemente reduzindo a população na área de cultivo. No que se refere à avaliação fitopatológica dos acessos de banco de germoplasma de figo, busca-se a identificação daqueles resistentes aos principais patógenos da figueira. Entretanto, estudos com avaliação do comportamento de acessos de figueira ao M. incognita são escassos. 
Para suprir a ausência de informações sobre materiais genéticos de figueira que sejam alternativos à cultivar 'Roxo de Valinhos', este trabalho objetivou caracterizar o desenvolvimento fenológico e avaliar a reprodução do nematoide das galhas $M$. incognita em diferentes acessos do Banco de Germoplasma de figueira.

\section{MATERIAL E MÉTODOS}

A avaliação fenológica foi realizada no período de agosto de 2012 a março de 2013 em acessos do Banco de Germoplasma da Figueira da Coordenadoria de Assistência Técnica Integral (CATI) que são mantidos no Núcleo de Produção de Mudas, em São Bento do Sapucaí, no Estado de São Paulo. A cidade está localizada a $22^{\circ} 41^{\prime}$ de latitude Sul e $45^{\circ} 44$ de longitude Oeste, a uma altitude média de 903 metros, possui clima classificado como Cwb subtropical de altitude, com temperaturas médias anuais próximas a $18^{\circ} \mathrm{C}$ e precipitação anual média entre 1.600 a $1.800 \mathrm{~mm}$ (Oliveira, 2013). As temperaturas médias registradas no período de avaliação fenológica estão apresentadas na Figura 1.

Figura 1. Valores médios de Temperatura $\left({ }^{\circ} \mathrm{C}\right)$ e precipitação $(\mathrm{mm})$ registrados durante o periodo de avaliação fenológica de diferentes acessos de figueira em São Bento do Sapucaí.

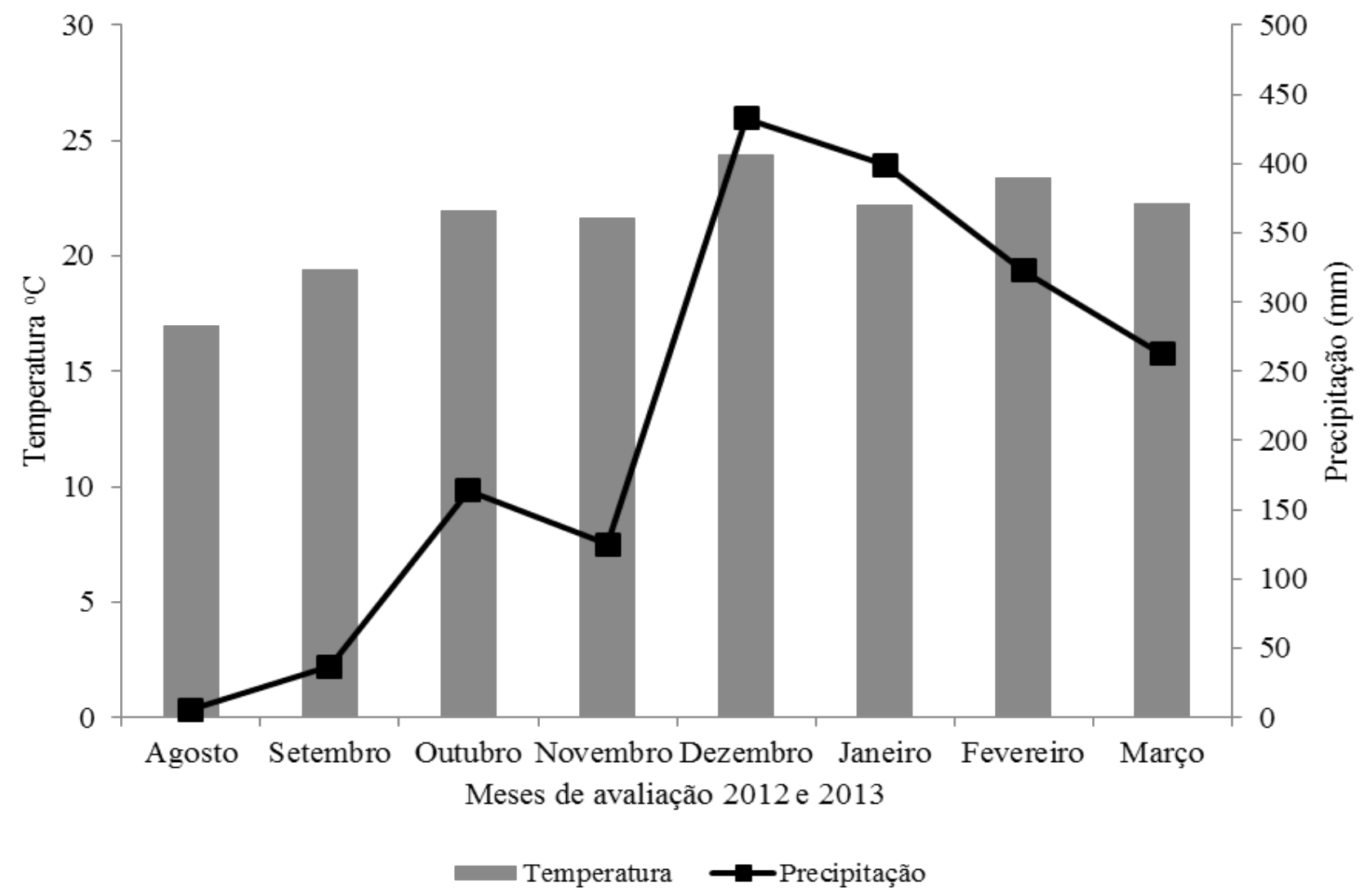

Foram avaliados 11 acessos do Banco de Germoplasma de figueira, com idade aproximada de cinco anos e denominados como: 'CATI II', 'CATI III', 'CATI IV', 'CATI V', 'CATI VI' E 'CATI VII' originados no Brasil e ainda 'Chile', 'Chile SN' e 'Chile 4/5' e 'Mission', procedentes do Chile e Estados Unidos, respectivamente. Esses acessos foram comparados a cultivar 'Roxo de Valinhos', principal cultivar plantada no Brasil. A área com os acessos foi dividida em quatro blocos e em cada um foram selecionadas quatro plantas de cada acesso. Nessas plantas foram selecionados quatro ramos e nesses, três gemas. A poda de inverno, prática de manejo comum à cultura da figueira, foi realizada em 01 de agosto de 2012, ocasião em que todas as plantas encontravam-se na fenofase gema de inverno ou gema dormente (Figura 2A), considerada estádio inicial. Após a poda foram avaliadas o inicio e fim das seguintes fases fenológicas: Pontas verdes (Figura 2B); alongamento do ramo (Figura 2C); desenvolvimento do fruto (Figura 2D) e maturação do fruto (Figura 2E). 
Figura 2. Fases fenológicas da figueira (Ficus carica) em São Bento do Sapucaí Gema de inverno ou gema dormente (A); Pontas verdes (B); Alongamento dos ramos (C); Desenvolvimento do fruto (D); Maturação do fruto (E).

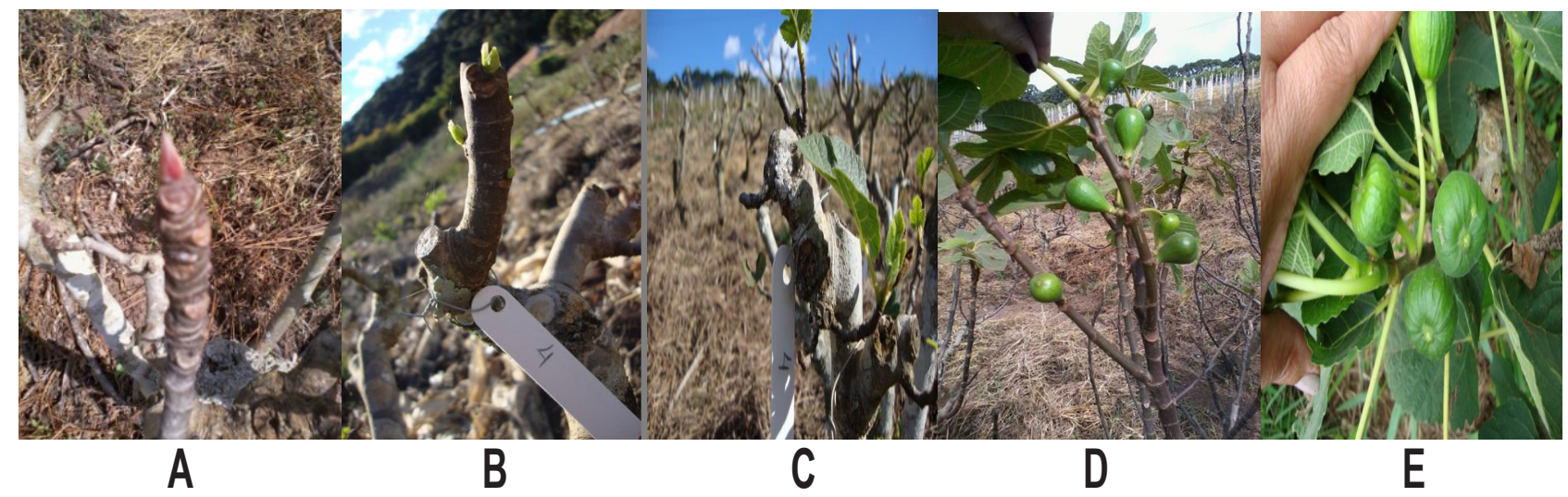

Para avaliação da hospedabilidade dos acessos ao $M$. incognita, foram formadas mudas a partir de estacas lenhosas, coletadas no Banco de germoplasma de figueira da CATI. Na Fazenda Experimental da Empresa de Pesquisa Agropecuária de Minas Gerais, Unidade Regional Sul de Minas, Lavras, essas estacas foram imersas em ácido indol butírico (AIB) $3000 \mathrm{mg} \mathrm{L}^{-1}$, em solução hidroalcoólica por 10 min e a seguir transferidas para caixas de areia até o enraizamento, quando então as mudas foram transplantadas para sacos de polietileno, com capacidade de 2,5L contendo substrato terra e areia (3:1) previamente esterilizado com dazomete (Basamid $^{\circledR}$ ) e mantidas em casa de vegetação.

Uma semana após o transplantio, as mudas foram inoculadas com aproximadamente 5700 ovos de M. incognita, distribuídos em dois orifícios próximos ao colo de cada muda. Esse inóculo, inicialmente oriundo de pomar da cv. Roxo de Valinhos, localizado no município de São Sebastião do Paraíso, Minas Gerais, e foi identificado conforme a técnica proposta por Carneiro e Almeida (2001), foi extraído por meio do método de Hussey e Barker (1973) das raízes de tomateiros CV. Santa Clara, empregados como multiplicadores do nematoide. A concentração dos ovos na suspensão do inóculo foi verificada em microscópio de objetiva invertida. 0 experimento foi instalado com onze tratamentos em delineamento de blocos casualizados com quatro repetições e duas plantas por parcela.

A avaliação da reprodução do $M$. incognita nos acessos foi realizada aos seis meses da inoculação e ocorreu no mês de outubro de 2012. Para tal, o sistema radicular das plantas foi cuidadosamente lavado, retirando o excesso de água pela deposição das raízes em papel absorvente e a seguir, obtido o peso da massa fresca total de raiz (g), PMFR. Posteriormente, o sistema radicular de cada planta foi cortado em fragmentos de aproximadamente dois centímetros e submetido ao método de extração de Hussey e Barker (1973). Foram quantificados os ovos e juvenis do segundo estádio (J2) empregando lâmina de contagem sob microscópio de objetiva invertida, em três leituras para obtenção do valor médio por amostra. Diante desses dados foi calculada a população total (ovos + Juvenis do segundo estádio - Ptotal) por grama de raízes e o fator de reprodução (FR) com base na relação entre a população final, dividida pela população inicial (Pf/Pi), conforme Cook \& Evans (1987), onde foram consideradas resistentes os acessos que apresentarem valor do FR $<1$ e suscetíveis aqueles com FR > 1. Os dados foram submetidos ao teste de Normalidade de Shapiro-Wilk e posteriormente analisados pelo programa estatístico $R$ ( $R$ Development Core Team, 2015) com auxílio do pacote ExpDes. As médias foram comparadas pelo teste Scott_Knott a 5\% de probabilidade.

\section{RESULTADOS E DISCUSSÃO}

Foi verificado efeito significativo entre os acessos em todas fenofases estudadas e os resultados obtidos para a avaliação dos estádios fenológicos (Tabela 1) mostram semelhança entre o comportamento em função da procedência dos acessos. A brotação foliar, caracterizada pelo enverdecimento da gema teve inicio, em média, 20 dias após a poda e no geral destaca-se o comportamento do acesso denominado 'Mission', que embora iniciando tardiamente esta fenofase inicial, apresentou menor comprimento do ciclo. Este fato pode ser atribuído ao vigor das 
plantas, notadamente pelo tamanho de suas folhas e corrobora com dados reportados por Vemmos et al. (2013) 0 acesso 'Mission', foi também o mais precoce na frutificação, estádio fenológico em que a diferença entre os acessos foi mais expressiva na maturação dos frutos, onde foram formados cinco grupos.

Tabela 1. Valores médios, em dias após a poda, correspondentes aos estádios fenológicos de figueira (Ficus carica) de diferentes acessos do Banco de Germoplasma durante ciclo vegetativo 2012-2013 em São Bento do Sapucaí: I) Pontas verdes; II) Alongamento dos ramos; III) Desenvolvimento do fruto; IV) Maturação do fruto e V) Comprimento do ciclo.

\begin{tabular}{l|c|c|c|c|c}
\hline \multirow{2}{*}{ Acessos } & \multicolumn{5}{|c}{ Fases fenológicas } \\
\cline { 2 - 6 } & $\mathrm{I}$ & $\mathrm{II}$ & III & IV & V \\
\hline Roxo de Valinhos & $35,95 \mathrm{a}^{*}$ & $10,74 \mathrm{~d}$ & $41,07 \mathrm{~d}$ & $57,44 \mathrm{~d}$ & $194,37 \mathrm{c}$ \\
Mission & $23,48 \mathrm{c}$ & $23,51 \mathrm{a}$ & $43,74 \mathrm{~d}$ & $50,03 \mathrm{e}$ & $174,51 \mathrm{~d}$ \\
Chile & $28,11 \mathrm{~b}$ & $18,62 \mathrm{c}$ & $61,22 \mathrm{c}$ & $70,77 \mathrm{c}$ & $251,51 \mathrm{a}$ \\
Chile s/n & $22,70 \mathrm{c}$ & $20,44 \mathrm{~b}$ & $77,85 \mathrm{a}$ & $65,44 \mathrm{c}$ & $264,29 \mathrm{a}$ \\
Chile 4/5 & $23,22 \mathrm{c}$ & $17,03 \mathrm{~b}$ & $78,29 \mathrm{~b}$ & $78,29 \mathrm{a}$ & $251,29 \mathrm{a}$ \\
CATI II & $26,96 \mathrm{~b}$ & $14,51 \mathrm{~b}$ & $73,37 \mathrm{c}$ & $73,37 \mathrm{~b}$ & $223,29 \mathrm{c}$ \\
CATI III & $23,00 \mathrm{~b}$ & $13,88 \mathrm{c}$ & $54,48 \mathrm{c}$ & $73,18 \mathrm{~b}$ & $219,03 \mathrm{c}$ \\
CATI IV & $30,29 \mathrm{~b}$ & $16,74 \mathrm{c}$ & $47,70 \mathrm{~d}$ & $73,55 \mathrm{~b}$ & $216,66 \mathrm{c}$ \\
CATIV & $29,59 \mathrm{~b}$ & $17,85 \mathrm{c}$ & $50,66 \mathrm{~d}$ & $71,37 \mathrm{~b}$ & $220,14 \mathrm{c}$ \\
CATI VI & $29,62 \mathrm{~b}$ & $19,07 \mathrm{~b}$ & $59,66 \mathrm{~b}$ & $69,25 \mathrm{c}$ & $237,29 \mathrm{~b}$ \\
CATI VII & $31,96 \mathrm{~b}$ & $20,22 \mathrm{~b}$ & $62,51 \mathrm{~b}$ & $67,81 \mathrm{c}$ & $246,29 \mathrm{~b}$ \\
Media Geral & 27,808 & 17,15 & 56,56 & 68,26 & 226,36 \\
\hline CV (\%) & 19,36 & 40,16 & 18,41 & 9,41 & 12,11 \\
\hline
\end{tabular}

*Médias seguidas da mesma letra na coluna não diferem entre si pelo teste de Scott_Knott a 5\% de probabilidade.

A influência do clima na duração do ciclo dos acessos foi positiva uma vez que a temperatura média registrada durante todo o período (Figura 1) esteve dentro da faixa considerada tolerável para a cultura, conforme reportado por Souza et al. (2009). A duração do ciclo teve seus valores médios oscilando entre 174 a 264 dias evidenciando a influência do clima na duração das fenofases, estando também associado à constituição genética de cada acesso.

Os acessos de figueira apresentaram diferença significativa $(P<0,05)$ quanto ao número de ovos $\mathrm{e}$ Juvenis do segundo estádio, sendo estatisticamente semelhante para o Fator de Reprodução, População total, número de galhas e peso da massa fresca de raízes (Tabela 2). De acordo com o peso da massa fresca do sistema radicular, verifica-se que todos os acessos apresentaram desenvolvimento radicular igual à cultivar Roxo de Valinhos. Alta reprodução do $M$. incognita, avaliada pelo Fator de Reprodução (FR), (Tabela 2), foi observada igualmente $(P<0,05)$ em todos os acessos de figueira e "Roxo de Valinhos", cultivar reconhecida em várias pesquisas, dentre elas Sherb et al. (1994), como altamente suscetível ao nematoide. A semelhança entre 0 número de galhas (NG) e a população total (ovos + Juvenis J2) por grama de raízes indica que os acessos de figo permitiram o estabelecimento e manutenção do sítio de alimentação com o desenvolvimento e reprodução do nematoide. Rezende et al. (2013) reporta que em cafeeiro pode ocorrer formação da galha e não ocorrer a reprodução do nematoide. Entretanto, no presente estudo verificou-se que todos os acessos de figueira estudados, embora de diferentes procedências, proporcionaram boa reprodução (Tabela 2), consequentemente boa e igual hospedabilidade ao M. incognita. Esses resultados confirmam ensaio realizado por Silva et al. (2012) onde os mesmos acessos foram avaliados em condições similares à deste estudo, porém com inoculação de 10.000 ovos de $M$. incognita por planta nesses acessos. 
Tabela 2. Caracterização de diferentes acessos provenientes do Banco de Germoplasma de figueira (Ficus carica) quanto à reprodução do Meloidogyne incognita verificada pelo número médio de Ovos, de Juvenis do segundo estádio (J2), pelo Fator de reprodução (FR), população total (ovos + J2, POP T) por grama de raiz extraída; número de galhas $(G)$ e Peso da Massa fresca do sistema radicular (PMFR) aos seis meses de inoculação. Lavras-MG, 2014.

\begin{tabular}{|c|c|c|c|c|c|c|}
\hline Acessos & Ovos & $\mathrm{J} 2$ & $\mathrm{FR}^{*}$ & POP T & Galhas & PMFR \\
\hline Roxo de Valinhos & $42.154,59 b^{* *}$ & $6.687,60 \mathrm{~b}$ & $9,21 \mathrm{a}$ & $48.842,50 a$ & $1.397,37$ & $114,29 a$ \\
\hline Mission & $43.438,5 b$ & $8.222,25 b$ & $9,74 a$ & $51.660,50 a$ & $1.722,65 a$ & $85,62 a$ \\
\hline Chile & $56.804,32 b$ & $14.813,54 a$ & $13,51 \mathrm{a}$ & $71.617,85 a$ & $1.491,75 a$ & $112,14 a$ \\
\hline Chile s/n & $54.235,63 b$ & $14.242,34 a$ & $12,44 a$ & $65.941,06 a$ & $1.324,75 a$ & $100,87 a$ \\
\hline Chile 4/5 & $57.787,19 b$ & $12.280,45 a$ & $13,22 a$ & $70.067,65 a$ & $1.418,00 a$ & $104,34 a$ \\
\hline CATI II & $35.171,86 b$ & $5.515,95 b$ & $7,67 a$ & $40.687,80 a$ & $1.097,37 a$ & $111,57 a$ \\
\hline CATI III & $77.840,73 a$ & $7.246,01 b$ & $16,05 a$ & $85.086,74 a$ & $1.245,12 \mathrm{a}$ & $101,19 a$ \\
\hline CATI IV & $95.814,85 a$ & $9.149,50 b$ & $19,80 a$ & $104.964,35 a$ & $1.871,00 \mathrm{a}$ & $115,96 a$ \\
\hline CATI V & $42.492,70 b$ & $4.353,37 \mathrm{~b}$ & $8,84 a$ & $468.46,07 a$ & $892,62 a$ & $96,41 \mathrm{a}$ \\
\hline CATI VI & $75.508,20 a$ & $12.095,96 \mathrm{a}$ & $16,52 a$ & $87.604,16 a$ & $1.346,25 \mathrm{a}$ & $96,73 a$ \\
\hline CATI VII & $43.330,10 a$ & $11.929,63 a$ & $10,42 a$ & $55.259,72 a$ & $1.739,87 a$ & $101,36 a$ \\
\hline Média Geral & 56779,87 & 9685,16 & 12,54 & 66465,03 & 1413,35 & 103,683 \\
\hline CV $(\%)$ & 45,06 & 42,57 & 42,36 & 42,36 & 39,15 & 14,21 \\
\hline
\end{tabular}

${ }^{*}$ FR=Pop Final/Pop inicial.

${ }^{* *}$ Médias seguidas da mesma letra na coluna não diferem entre si pelo teste de Scott_Knott a 5\% de probabilidade.

Avaliando a diversidade genética desses acessos de figueira com 0 uso de marcadores microssatélites, Val et al. (2013) verificaram que os acessos 'Chile' e 'Chile SN' são indivíduos semelhantes, e que os demais apresentaram ampla diversidade genética entre si. Entretanto, esta diversidade não influenciou a hospedabilidade das plantas ao nematoide, visto que a população de ovos e juvenis de $M$. incognita foi estatisticamente igual em todos os acessos avaliados no presente estudo. Ressalta-se que este resultado pode estar associado ao desenvolvimento do sistema radicular das plantas uma vez que este foi determinante na definição da época de avaliação em decorrência do enovelamento das raízes.

\section{REFERÊNCIAS BIBLIOGRÁFICAS}

CARNEIRO, R. M. D. G.; ALMEIDA, M. R. A. Técnica de eletroforese usada no estudo de enzimas dos nematoides de galhas para identificação de espécies. Nematologia Brasileira, 2001, 25, 1, 35-44.

COOK, C.G.; EVANS, K. RESISTANCE AND TOLERANCE. IN: BROWN, R.H.; KERRY, B.R. (Ed.). Principles and practice of nematode control in crops. Orlando: Academic Press, 1987. p.179-231.
DIAS-ARIEIRA, R.C.; FURLANETTO, C.; SANTANA, S.M.; BARIZÃO, D. A. O.; RIBEIRO, R. C.F.; FORMENTINI, H. M. Fitonematoides associados a frutíferas na região noroeste do estado do Paraná, Brasil. Revista Brasileira de Fruticultura, 2010, 32, 4, 1064-1071.

FAO. Food Agricultural Organization. Statistical database, 2012. Disponível em: <http://faostat.fao.org/ site/339/default.aspx>. Acesso em 14 outubro 2014.

FACHINELLO, J. C.; PASA, M. S.; SCHMTIZ, J. D.; BETEMPS, D. L. Situação e perspectivas da fruticultura de clima temperado no Brasil. Revista Brasileira de Fruticultura, 2011, 33, 1, 109-120.

FERREIRA, E. A.; PASQUAL, M.; TULMANN NETO, A. In vitro sensitivity of fig plantlets to gamma rays. Scientia Agricola, 2009, 66, 540-542.

GOMES, C. B.; SOMAVILLA, L.; CARNEIRO, R.M.D.G.; COSTA, F.A.; MEDINA, I.L. Monitoramento do nematóide das galhas (Meloidogyne spp.) em figueira (Ficus carica L.) no Rio Grande do Sul. Pelotas: Embrapa Clima Temperado Boletim de pesquisa e desenvolvimento, n. 86, 2009, 18p.

GONÇALVES, C. A. A.; LIMA, L. C. O.; LOPES, P. S. 
N.; SOUZA, M. T. Poda e sistemas de condução na produção de figos verdes. Pesquisa Agropecuária Brasileira, 2006, 41, 6, 955-961.

HUSSEY, R. S.; BARKER, K. R. A comparison of methods of collecting inocula of Meloidogyne spp. including a new technique. Plant Disease Reporter, $1973,57,12,1025-1028$.

IBGE - Instituto Brasileiro de Geografia e Estatística. Produção agrícola municipal. Disponível em: <http:/l www.ibge.gov.br>. Acesso em: 7 outubro 2010.

LARCHER, W. Ecofisiologia vegetal. São Carlos: Rima, 2000. $531 \mathrm{p}$.

LIETH, H. Introduction to phenology and the modeling of seasonality. Phenology and seasonality modeling. In Ecological Studies 8. (H. Lieth, ed.). Springer-Verlag, Berlin, p.3-19, 1974.

MAIORANO, J. A.; ANTUNES, L. E. C.; REGINA, M. A.; ABRAHÃO, E.; PEREIRA, A. F. Botânica e caracterização de cultivares de figueira. Informe Agropecuário, 1997, 18, 188, 22-24.

MEDINA, I. L.; SOMAVILLA, L.; CARNEIRO, R. M. D. G.; GOMES, C. B. Espécies de Meloidogyne em figueira (Ficus carica) e em plantas infestantes. Nematropica, 2013, 43, 1, 56-62.

MEDINA, I. L.; GOMES, C. B.; ROSSI, C.; CARNEIRO, R. M. D. G. Caracterização e Identificação de Populações de Nematóides de Galhas Provenientes de Figueiras (Ficus carica L.) do Rio Grande do Sul e de São Paulo. Nematologia Brasileira, 2006, 30, 2, 179187.

MOURA, R. M. O gênero Meloidogyne e a meloidoginose - Parte II. Revisão Anual de Patologia de Plantas, 1997, 5, 281-315.

NIENOW, A. A.; CHAVES, A.; LAJÚS, C. R.; CALVETE, E. O. Produção da figueira em ambiente protegido submetida a diferentes épocas de poda e número de ramos. Revista Brasileira de Fruticultura, 2006, 25, 3, 421-424.

OLIVEIRA, M.C.; PIO, R. RAMOS,J.D.; LIMA, L.C.O.; PASQUAL,M. SANTOS, V.A. Fenologia e características físico-químicas de frutos de abacateiros visando à extração de óleo. Ciência Rural, 2013, 43, 3,411-418.

R DEVELOPMENT CORE TEAM, R: A Language and Environment for Statistical Computing. Vienna, Austria: the R Foundation for Statistical Computing. ISBN: 3-900051-07-0. Available online at http://www.Rproject.org/. Acesso em 7 de outubro de 2015.

REZENDE, R. M.; SALGADO, S. M. L.; REZENDE, J. C.; CARVALHO, G. R.; PEREIRA, A. A.; LIMA, R. R.; FERREIRA, A. D. Resistência de progênies de Coffea arabica em área infestada por Meloidogyne exigua. Nematropica, 2013, 43, 2, 233-240.

SHERB, C.T.; CAMPOS, V. P.; CHALFUN, N. N. J. Flutuação populacional de Meloidogyne incognita em figueiras inoculadas no campo. Nematologia Brasileira, 1994, 18, 1, 14-15.

SILVA, E. A.; FERREIRA, E. A.; SALGADO, S. M. L.; SOUZA, S. R.; FLORENCIO, V.H. R.; CARVALHO, R. D. Avaliação da patogenicidade de Meloidogyne incognita a genótipos de Ficus carica. In: XXII CONGRESSO BRASILEIRO DE FRUTICULTURA, 2012, Bento Gonçalves, Anais do XXII CONGRESSO BRASILEIRO DE FRUTICULTURA, Bento Gonçalves, 2012.

SOUZA, A.P.; SILVA, A. C.; LEONEL, S.; ESCOBEDO, J. F. Temperaturas basais e soma térmica para a figueira podada em diferentes épocas. Revista Brasileira de Fruticultura, 2009, 31, 2, 314-322.

VAL, A. D. B.; SOUSA, C. S.; FERREIRA, E. A.; SALGADO, S. M.; PASQUAL, M.; CANÇADO, G. M. A. Evaluation of genetic diversity in fig accessions by using microsatellite markers. Genetics and Molecular Research, 2013, 12, 2, 1383-1391.

VEMMOS, S.N.; PETRI, E.; STOURNARAS, V. Seasonal changes in photosynthetic activity and carbohydrate content in leaves and fruit of three fig cultivars (Ficus carica L.) Scientia Horticulturae, 2013, 160, 198-207. 